\title{
EMITTANCE GROWTH DUE TO NOISE AND ITS SUPPRESSION WITH THE FEEDBACK SYSTEM IN LARGE HADRON COLLIDERS
}

\author{
V. LEBEDEV, ${ }^{*}$ V. PARKHOMCHUK, ${ }^{*}$ V. SHILTSEV,${ }^{*}$ and G. STUPAKOV
}

Superconducting Super Collider Laboratory ${ }^{\dagger}$

2550 Beckleymeade Ave. Dallas, TX 75237

(Received 28 April 1993; in final form 10 February 1994)

\begin{abstract}
The problem of emittance growth due to random fluctuation of the magnetic field in hadron colliders is considered. Based on a simple one-dimensional linear model, a formula for emittance growth rate as a function of the noise spectrum is derived. Different sources of the noise are analyzed and their role is estimated for the Superconducting Super Collider (SSC). A theory of feedback suppression of the emittance growth is developed which predicts the residual growth of the emittance in the accelerator with a feedback system.
\end{abstract}

KEY WORDS: Beam cooling, emittance growth, noise, hadron colliders, feedback, particle dynamics, storage rings

\section{INTRODUCTION}

Future large hadron colliders such as LHC and especially the Superconducting Super Collider (SSC) will have a circumference of tens of kilometers. That means that the revolution frequency in these machines comes into the range of several kilohertz. Typically, the level of the external noise (such as ground motion, current ripple, etc.) increases when one goes to lower frequencies, so noise effects that probably were not an issue for smaller storage rings might seriously degrade performance of large machines. For the proton colliders with beam energy of tens of $\mathrm{TeV}$, the synchrotron radiation is still not strong enough to counteract the noise effects.

Depending on the frequency of the noise, we can distinguish two mechanisms of the beam perturbation. At low frequencies (much less than the revolution frequency), the noise produces a distortion of the closed orbit of the beam. For the SSC, these

\footnotetext{
* Visiting from the Budker Institute of Nuclear Physics, Novosibirsk, Russia.

$\dagger$ Operated by the Universities Research Association, Inc., for the U.S. Department of Energy under Contract No. DE-AC35-89ER40486.
} 
effects have been previously considered in References $1-5$. However, if the spectrum of the noise extends up to the resonant betatron frequencies $f_{0}|\nu-n|$, where $f_{0}$ is the revolution frequency, $\nu$ is the tune and $n$ is an integer, it resonantly drives the betatron oscillations of the beam. Due to decoherence, these oscillations rapidly translate into the growing emittance of the beam with a growth rate that is proportional to the noise spectrum at the resonant frequency. ${ }^{6-9}$

An effective way to suppress the emittance growth caused by the noise is based on the use of a feedback system that monitors the amplitude of the betatron oscillations and tries to damp them by applying appropriate kicks to the beam. If the feedback system damps beam oscillations faster than they decohere, the emittance growth will be strongly suppressed compared to the case without feedback.

In this paper, we first address the problem of emittance growth due to random fluctuation of the dipole magnetic field B. Using simple one-dimensional linear model, in Section 2 we derive a formula for an emittance growth rate as a function of the noise spectrum. In Section 3, we analyze different sources of the noise and estimate their role for the SSC. For completeness, in this section we also included results of the consideration of the emittance growth due to the fluctuation of the gradient of the magnetic field $B^{\prime}$. In Section 4, we develop a theory of feedback suppression of the emittance growth and calculate the residual growth of the emittance in the accelerator with a feedback.

In a subsequent paper, we will present results of computer simulations that confirm the predictions of the analytical theory of this paper and also take into account additional effects not covered by the simple theory used in the present paper.

\section{EMITTANCE GROWTH DUE TO EXTERNAL NOISE}

\subsection{General Considerations}

Considering particle motion in an accelerator, we will neglect coupling between vertical and horizontal degrees of freedom and will use the following variables:

$$
x=\frac{X}{\sqrt{\beta}}, \quad p=\beta \frac{d}{d s} \frac{X}{\sqrt{\beta}},
$$

where $X$ stands for the particle deviation with respect to the closed orbit, $\beta$ is the beta function, and $s$ is the path length along the orbit.

Assume that the magnetic field in one of the magnets is perturbed by an amount $\delta B(t)$ and varies with time. This perturbation may be due to random (time-dependent) displacements of a quadrupole or fluctuations of the current in a dipole magnet.

Each turn when a particle passes through the magnet, the particle experiences a kick that changes its momentum from $p$ to $p^{\prime}$,

$$
p^{\prime}=p+\Delta p_{n}
$$


where the change of the momentum $\Delta p_{n}$ is related to the perturbation of the magnetic field,

$$
\Delta p_{n}=\sqrt{\beta_{0}} \frac{e l \delta B(n T)}{P c} .
$$

In Equation (3), $P$ is the particle longitudinal momentum, $l$ is the length of the magnet, $\beta_{0}$ is the beta function at the position of the magnet, $T$ is the revolution period, and $n$ is the turn number. Taking into account that free betatron oscillations are described by the following transformation of the variables $x$ and $p$,

$$
x^{\prime}=x \cos \theta+p \sin , \theta, p^{\prime}=-x \sin \theta+p \cos \theta,
$$

where $\theta$ stands for the betatron phase, we can write down the result of $N$ successive passes through the magnet.

$$
x_{N}=\sum_{n=0}^{N-1} \Delta p_{n} \sin \mu(N-n)+x_{0} \cos \left(\mu N+\theta_{0}\right),
$$

where $\mu=2 \pi \nu, \nu$ is the tune and $x_{0}$ and $\theta_{0}$ are the initial amplitude and phase of the oscillations.

Throughout this paper, we will be assuming that $\delta B(t)$ is a stationary random function characterized by its correlation function $K_{\delta B}(\tau)$,

$$
K_{\delta B}(\tau)=\langle\delta B(t) \delta B(t-\tau)\rangle,
$$

where the angle brackets denote the averaging. Using Equation (3), we will also define the correlation function of the kicks,

$$
K_{\Delta p}(\tau)=\langle\Delta p(t) \Delta p(t-\tau)\rangle=\beta_{0}\left(\frac{e l}{P c}\right)^{2} K_{\delta B}(\tau)
$$

so that

$$
\left\langle\Delta p_{n} \Delta p_{m}\right\rangle=K_{\Delta p}(T(n-m)) .
$$

Related to the correlation function $K(\tau)$ is the corresponding spectral density (or, briefly, spectrum), $S(\omega)$. It is a positive even function of the frequency $\omega: S(\omega) \geq$ $0, S(\omega)=S(-\omega)$. According to the Wiener-Khintschin theorem, the spectrum can be found as a Fourier transform of the correlation function:

$$
K(\tau)=\int_{-\infty}^{\infty} d \omega S(\omega) e^{-i \omega \tau}=2 \int_{0}^{\infty} d \omega S(\omega) \cos \omega \tau, \quad S(\omega)=\frac{1}{2 \pi} \int_{-\infty}^{\infty} d \tau K(\tau) e^{i \omega \tau}
$$

Note that a frequently used in many publications concept of white noise corresponds to the noise spectrum that does not depend on the frequency, i.e., that $S(\omega)=$ constant. 


\subsection{Growth of the Amplitude of the Betatron Oscillations}

Using Equation (5) we can now calculate the averaged square of $x_{N},\left\langle x_{N}^{2}\right\rangle$. In doing so, we will utilize the condition ${ }^{a}\left\langle\Delta p_{n}\right\rangle=0$,

$$
\left\langle x_{N}^{2}\right\rangle=x_{0}^{2} \cos ^{2}\left(\mu N+\theta_{0}\right)+\sum_{n, m=0}^{N-1} K_{\Delta p}(T(n-m)) \sin \mu(N-n) \sin \mu(N-m) .
$$

To facilitate the summation in Equation (10), we express $K_{\Delta p}$ in terms of the spectral density $S_{\Delta p}$ using Equation (9),

$$
\left\langle x_{N}^{2}\right\rangle=x_{0}^{2} \cos ^{2}\left(\mu N+\theta_{0}\right)+\frac{1}{2} \int_{-\infty}^{\infty} S_{\Delta p}(\omega) \sum_{n, m=0}^{N-1} e^{i \omega T(n-m)} \sin \mu(N-m) \sin \mu(N-m) .
$$

The summation in Equation (11) can now be performed explicitly. We are interested here in the limit of large $N$, formally $N \rightarrow \infty$. As shown in the Appendix, in this limit Equation (11) reduces to

$$
\left\langle x_{N}^{2}\right\rangle=\frac{1}{2} \sum(\nu) N \Omega+x_{0}^{2} \cos ^{2}\left(\mu N+\Theta_{0}\right),
$$

where $\Omega$ is the revolution frequency, $\Omega=2 \pi / T$, and

$$
\sum(\nu)=\sum_{n=-\infty}^{\infty} S_{\Delta p}((\nu-n) \Omega)=\beta_{0}\left(\frac{e l}{P c}\right)^{2} \sum_{n=-\infty}^{\infty} S_{\delta B}((\nu-n) \Omega) .
$$

A special discussion is needed if one applies Equation (13) to the white noise. Formally, putting $S(\omega)=$ constant into Equation (13) gives infinity on the right-hand side. This happens because the usual definition of the white noise assumes that it has the correlation function $\propto \delta(\tau)$ and, hence, an infinite value of $\left\langle\delta B^{2}\right\rangle$. For our problem, it is more convenient to change this definition so as to understand white noise as having a correlation time much less than the revolution period. In other words, the white noise in a magnet produces uncorrelated kicks on the beam, $\left\langle\Delta p_{n} \Delta p_{m}\right\rangle=0$ if $n \neq m$, and

$$
K_{\Delta p}(T(n-m))=\left\langle\Delta p_{n}^{2}\right\rangle \delta_{n, m},
$$

where $\delta_{n, m}$ is the Kroneker symbol. Putting this equation into Equation (10) and performing the summation, one easily arrives at Equation (12) with the following $\sum(\nu)$ :

$$
\sum(\nu)=\frac{1}{\Omega}\left\langle\Delta p_{n}^{2}\right\rangle=\beta_{0}\left(\frac{e l}{P c}\right)^{2} \frac{1}{\Omega}\left\langle\delta B^{2}\right\rangle
$$

\footnotetext{
${ }^{a}$ This condition can always be met by proper choice of equilibrium closed orbit.
} 
From Equation (13) it follows that, due to the presence of the noise, the average square of the single-particle displacement grows linearly with time. This time dependence is typical for diffusion processes, in our case the diffusing quantity being the amplitude of the betatron oscillations. The only spectral components that contribute to the growth of the amplitude have a frequency equal to that of the betatron sidebands. For the SSC, ${ }^{10}$ the revolution frequency $f_{0}=\Omega / 2 \pi=3.4 \mathrm{kHz}$ and the nominal fractional part of the tune, $\{\nu\}$, is equal to 0.28 . Thus the lowest resonant frequency of the noise is equal to $\{\nu\} f_{0}=960 \mathrm{~Hz}$.

\subsection{Tune Spread and the Emittance Growth}

In the above derivation, the motion of only one particle of the beam has been considered. Since the first term on the right-hand side of Equation (5) does not depend on the particle's initial amplitude and phase, random dipole kicks will drive coherent oscillations of the beam as a whole. However, the tune spread in the beam causes phase mixing of different particles and makes the betatron oscillations lose coherence on a time scale equal to the inverse spread of the betatron frequencies.

There are several sources of tune spread in hadron accelerators. First, because of the finite energy spread of the beam, it can stem from the chromaticity of the machine. Second, the tune spread is generated by nonlinear elements in the lattice, such as sextupoles and octupoles, as well as higher-order multipole fields in the various magnets. Finally, in colliders, the dominant contribution to the tune spread usually comes from the collisions themselves; we will assume this for the sake of further discussion. In this case, for round beams and Gaussian particle distribution function, the rms tune spread can then be related to the "interaction parameter" $\xi$,

$$
\xi=\frac{e^{2} N_{\text {particle }}}{4 \pi P c \varepsilon}
$$

which, in the limit $\xi \ll 1$, coincides with the betatron frequency shift. In accordance with Reference 8 , the rms betatron frequency spread is equal to

$$
\Delta \nu_{\mathrm{rms}} \equiv \sqrt{\overline{\overline{\Delta \nu^{2}}}} \approx 0.2 \xi,
$$

where the bar denotes averaging over the particle distribution function. In Equation (16), $N_{\text {particle }}$ is the number of particles in the bunch and $\varepsilon$ is the beam emittance. For the SSC, $\xi=1.8 \times 10^{3}$ for two interaction regions, and twice that if four interaction regions will be operating. This gives a decoherence time $\tau_{\text {decoh }}=1 / 2 \pi f_{0} \Delta \nu_{\mathrm{rms}} \approx$ $0.1-0.06 s$.

Decoherence rapidly translates dipole beam oscillations driven by external noise into emittance growth. In accordance with the standard definition of beam emittance $\varepsilon$, it is equal to the square of the offset $\overline{x^{2}}$ averaged over the particle distribution function of the beam. ${ }^{b}$ With $\varepsilon$ so defined one can rewrite Equation (12) in terms of emittance:

\footnotetext{
${ }^{b}$ This definition of emittance is equivalent to $\varepsilon=\frac{1}{2}\left(\overline{p^{2}}+\overline{x^{2}}\right)$, because in our case $\overline{x^{2}}=\overline{p^{2}}$.
} 


$$
\langle\varepsilon(t)\rangle=\frac{t}{4 \pi} \sum(\nu) \Omega^{2}+\varepsilon(0) .
$$

An important characteristic of the noise effect is the derivative $d\langle\varepsilon\rangle / d t$ which gives the rate of the emittance growth:

$$
\left(\frac{d\langle\varepsilon\rangle}{d t}\right)_{0}=\frac{1}{4 \pi} \sum(\nu) \Omega^{2}
$$

We put the index 0 on the left-hand side of Equation (19) to emphasize that this growth of emittance occurs without a feedback system in the machine.

Two remarks should be made in connection with Equation (19). First, as follows from Equation (13), only the noise at a discrete set of frequencies contributes to the emittance growth. However, due to the betatron frequency spread in the beam, the resonant frequency of different particles deviates from the frequency $(n-\nu) \Omega$, resulting in a finite resonance width $\Delta \omega_{\text {res }} \approx \Delta \nu \Omega$. Hence, more rigorously, rather than having exactly the frequency $(n-\nu) \Omega$, the resonant noise occupies the frequency range $\Delta \omega_{\text {res }}$ in the vicinity of this value. One can neglect the resonance width and use Equations (19) and (13) if the noise spectrum does not change appreciably within the width of the resonance $\Delta \omega_{\text {res }}$.

Second, it turns out that the above derivation is valid only if the tune is not too close to nonlinear resonances of the machine. Analytical study of these resonances in the presence of the external noise is a cumbersome problem. We will address this issue in a subsequent paper devoted to results of computer simulation of the emittance growth.

\section{SOURCES OF EXTERNAL NOISE AND ESTIMATES OF THEIR EFFECT}

The main noise sources in accelerators that produce transverse kicks on the beam are quadrupole vibrations and fluctuations of the magnetic field in the bending magnets and kickers. In this section, we will estimate a tolerable level of the noise in a collider without a feedback system.

\subsection{Vibrations of Quadrupoles}

Quadrupole vibrations are caused by both ambient seismic ground motion and manmade noise that is inevitably generated on the machine site by flows of coolant, operating vacuum pumps, etc. In many cases, it is impossible to trace the exact origin of these vibrations. Measurements in different $\operatorname{sites}^{11-14}$ show that the amplitude and spectrum of the ground motion may differ by several orders of magnitude depending on local conditions of the site and the time of day. Some examples of such spectra are shown in Figure 1. It clearly demonstrates that the spectral density of the ground motion strongly decreases with frequency. This feature explains why the noise effects are typically negligible for small rings but might be important for machines with a low revolution frequency such as the SSC and LHC. 


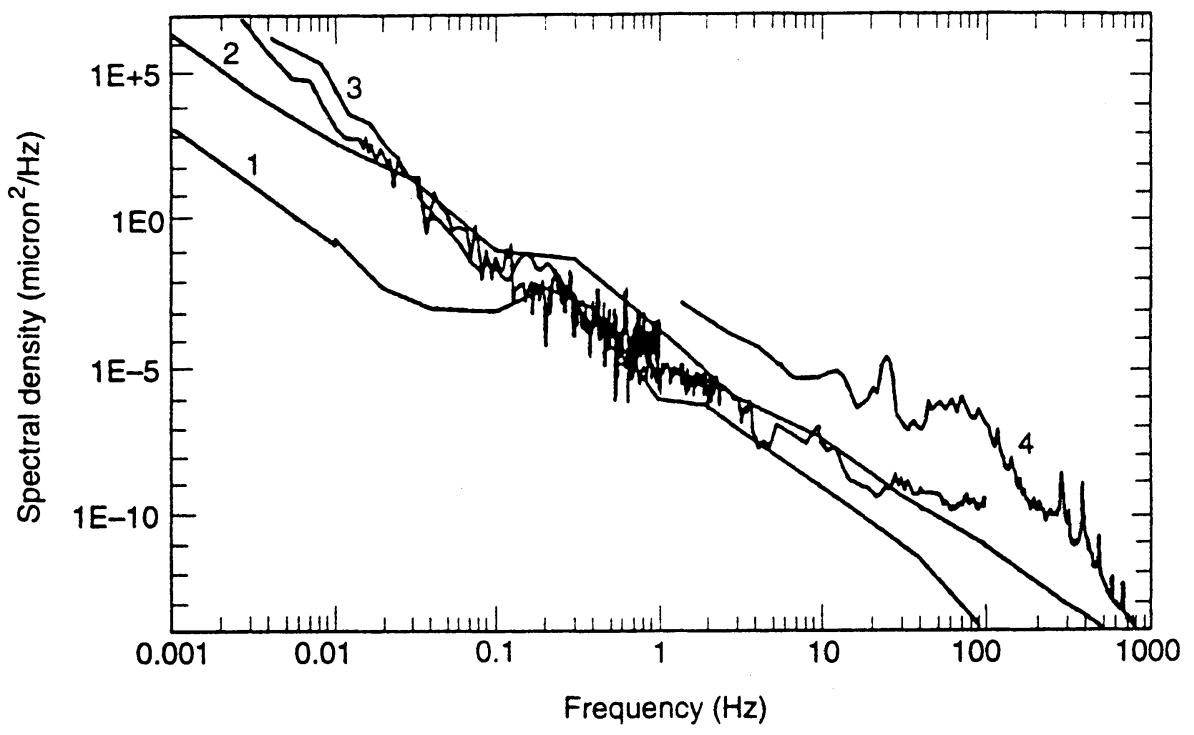

FIGURE 1: Spectra of ground motion at different sites: 1-Ref. 11; 2-Ref. 12; 3-Ref. 1; 4-Ref. 13.

To estimate the emittance growth arising from random vibrations of the quadrupoles, note that in this case, $\delta B$ is proportional to the average over the length of the magnet displacement of the quadrupole $d$,

$$
\delta B(t)=B^{\prime} d(t),
$$

where $B^{\prime}$ stands for the gradient of the magnetic field in the quadrupole. Using the definition of the focal length $f_{q}$, of the quadrupole $f_{q}=c P / e l B^{\prime}$, one can easily express $\sum(\nu)$ in terms of the spectrum of the displacement $S_{d}(\omega)$,

$$
\sum(\nu)=\frac{\beta_{0}}{f_{g}^{2}} \sum_{n=-\infty}^{\infty} S_{d}((\nu-n) \Omega) .
$$

To obtain a rough estimate of a tolerable level of magnet vibration, we assume that the vibration spectrum is the same for all of the quadrupoles and there is no correlation between displacements of the different quadrupoles in the ring (this is a reasonable assumption, considering that the relevant frequencies are in the range of a kilohertz and above). Let us also assume a focusing-defocusing as FODO lattice so that the quadrupoles are located at the positions of maximum and minimum values of the beta function. Summing Equation (21) over all quadrupoles of the ring, one finds

$$
\sum(\nu)=N_{\text {cells }} \frac{\beta_{\max }+\beta_{\min }}{f_{q}^{2}} \sum_{n=-\infty}^{\infty} S_{d}((\nu-n) \Omega)
$$


where $N_{\text {cell }}$ is the total number of cells and $\beta_{\max }, \beta_{\min }$ are the maximum and minimum values of the beta function in the cell. Note also that for the FODO lattice, $\left(\beta_{\min }+\right.$ $\left.\beta_{\min }\right) / f_{q}^{2}=8 \tan (\Delta \mu / 2) / L$, where $\Delta \mu$ is the phase advance through a full FODO period and $L$ is the length of the half cell. For the SSC, $\Delta \mu=90^{\circ}, L=90 \mathrm{~m}$, $N_{\text {cells }}=392$ (the number of cells in the arcs), and the nominal value of the emittance $\varepsilon=4.7 \times 10^{-9} \mathrm{~cm}$. Requiring that the emittance doubling time be larger than 20 hours (that is, approximately twice the synchrotron radiation cooling time), one finds the following limitation on the noise spectrum:

$$
\sum_{n=-\infty}^{\infty} S_{d}((\nu-n) \Omega) \leq 6 \times 10^{-13} \mu m^{2} / H z
$$

For comparison, note that for the white noise, this level of vibration corresponds to the rms quadrupole displacement $d_{\mathrm{rms}}=1.1 \times 10^{-4} \mu \mathrm{m}$.

Since the noise spectra falls rapidly with the frequency, the dominant term on the left-hand side of Equation (23) is the one with the lowest frequency. Again, for the nominal tune in the SSC, this frequency is equal to $960 \mathrm{~Hz}$ and Equation (23), in fact, gives an upper limit for the noise at this frequency.

In the kilohertz range of frequencies, a quadrupole several meter long does not oscillate as a rigid body, but rather experiences bending and torquing deformations around its axes. If the wavelength of such deformations is much smaller than the length of the magnet, the perturbation of the magnetic field averaged along the orbit is suppressed in comparison with the case where the magnet displaces as a whole. In other words, the displacement $d$ in Equation (20) becomes effectively smaller than the amplitude of the ground motion. However, analysis indicates ${ }^{15}$ that such a suppression is counteracted by multiple mechanical resonances of the magnet body, which can significantly amplify the vibrations at the resonant frequencies of the magnet.

Note also another specific mechanism of magnetic-field perturbation that comes into play if the vacuum tube has an inner liner with sufficiently high conductivity. Since high-frequency perturbations of the magnetic field are "frozen" in the liner, its vibrations will perturb the magnetic field on the orbit, even if the quadrupole itself is at rest.

\subsection{Dipole Field Fluctuations}

The effect of the bending magnetic field fluctuations can be easily estimated by using Equation (13) for the function $\sum$ and assuming that the fluctuations are independent and have the same spectrum in all of the bending magnets. For the sake of simplicity, we will also assume that these fluctuations are represented by white noise, and will use Equation (15). For the SSC parameters, with the total number of bending magnets $N_{\text {magnet }}=4200$ and the requirement that the emittance doubling time be less than 20 hours, this gives the following tolerable level of fluctuations in a magnet:

$$
\frac{\delta B_{\mathrm{rms}}}{B} \leq 6.9 \times 10^{-10}
$$


Note that magnetic-field fluctuation will be somewhat suppressed by the skin effect in the walls of the vacuum chamber (and/or liner), which can prevent the ac magneticfield component from penetrating the chamber. For the SSC, attenuation due to this effect is expected to be about ten- to twenty-fold which substantially loosens the tolerances for current fluctuation in the dipoles. On the other hand, the "freezing" of the magnetic field into the beampipe walls might also have a detrimental effect as a result of vibration modes that change the shape of the beampipe cross section (e.g., making the cross section elliptical rather than circular). Such modes perturb the dipole field inside the beam pipe, modulating it with the frequency of the vibrations.

To avoid confusion, we have to emphasize here that magnetic-field fluctuations can also be produced by power-supply ripple. However, in contrast to a wideband noise, the main components of the ripple are usually concentrated at several well-defined frequencies, and one can, in principle, avoid their detrimental influence by detuning the working point of the machine away from these frequencies. On the other hand, any random noise in the ripple will add to the perturbation of the magnetic field caused by other sources.

\subsection{Quadrupole Field Fluctuations and Vibrations of Sextupoles}

We have considered fluctuations of the dipole magnetic field in the beam orbit. The time-dependent perturbations of higher-order magnetic multipoles can also blow up the beam emittance. For the quadrupole field perturbation that can be generated either by vibration of the sextupoles or current fluctuations in the quadrupoles, the problem has been studied in References 16 and 17 . Here we present the results of these papers and estimate the effect for the SSC.

For a Gaussian density distribution of the beam, the emittance growth rate caused by random fluctuations of the gradient of the magnetic field in a magnet of length $l$ is given by ${ }^{17}$

$$
\frac{d\langle\varepsilon\rangle}{d t}=\frac{\varepsilon}{2 \pi} \Omega^{2}\left(\frac{l \beta_{0} e}{P c}\right)^{2} \sum_{n=-\infty}^{\infty} S_{B^{\prime}}((2 \nu-n) \Omega),
$$

where $\beta_{0}$ is the beta function at the position of the magnet and $S_{B^{\prime}}$ is the spectral density of the fluctuation of the gradient of the magnetic field $B^{\prime}$.

Note that, in contrast with Equation (13), resonant frequencies that contribute to the emittance growth are the sidebands of the double betatron frequency. This feature has a simple physical explanation: fluctuations of the quadrupole field bring about modulation of the tune and make the particle motion unstable via the parametric resonance. As is known, this resonance occurs at twice the frequency of the oscillator.

One can use Equation (25) to make an estimate of a tolerable level of fluctuations of the gradient of the magnetic field in quadrupoles. For the SSC, we assume white noise and take into account that the number of quadrupoles in the ring is $N_{\text {quad }} \approx 800$, each of them having a focal length $f_{q} \approx 60 \mathrm{~m}$. Half of the quadrupoles are located at the 
positions with a local maximum of $\beta, \beta_{0}=305 \mathrm{~m}$, giving the dominant contribution to the emittance growth. Again, requiring the emittance doubling time to be larger than 20 hours, one finds, from Equation (25):

$$
\frac{\delta B_{\mathrm{rms}}^{\prime}}{B^{\prime}} \leq 6 \times 10^{-7} \text {. }
$$

The gradient of the magnetic field on the orbit can also be perturbed by vibrations of the sextupole magnets. As a feed-down effect of an offset of a sextupole by some distance $d$, one finds the following perturbation of the gradient of the magnetic field:

$$
\delta B^{\prime}=B^{\prime \prime} d .
$$

Using Equation (27), one can rewrite Equation (24) in terms of the spectral density of the vibrations,

$$
\frac{d \varepsilon}{d t}=\frac{\varepsilon}{8 \pi} \Omega^{2}\left(\frac{B^{\prime \prime} l \beta_{0} e}{P c}\right)^{2} \sum_{n=-\infty}^{\infty} S_{d}((2 \nu-n) \Omega) .
$$

For the SSC, according to collider specifications, the product $B^{\prime \prime} l$ for about 400 sextupoles located near the focusing quadrupoles is equal to $2.4 \times 10^{3} \mathrm{~T} / \mathrm{m}$. Being located at the positions with the local maximum of the beta function, $\beta_{0}=305 \mathrm{~m}$, these sextupoles make the dominant contribution to Equation (28). Taking the nominal collider parameter $P c=20 \mathrm{TeV}$ and requiring the emittance doubling time to be more that 20 hours, one finds,

$$
\sum_{n=-\infty}^{\infty} S_{d}((2 \nu-n) \Omega) \leq 1.5 \times 10^{-5} \mu m^{2} / H z .
$$

As was mentioned above, for a rapidly decreasing noise spectrum, the dominant term on the left-hand side of Equation (29) is the one with the lowest frequency. For the nominal tune in the SSC, this frequency is equal to $1.52 \mathrm{kHz}$, and Equation (29) gives, in fact, an upper limit for the noise level at this frequency. Comparing it with Equation (23), we notice that this limitation is much less stringent than that originating from the quadrupole vibrations.

\section{EMITTANCE GROWTH SUPPRESSION WITH A FEEDBACK SYSTEM}

\subsection{Basics of the Feedback Theory}

A transverse feedback system allows one to suppress the emittance growth caused by excitation of the betatron oscillations by external noise. The system monitors the offset of the beam centroid and tries to correct it by kicks, proportional to this offset, applied a quarter of the betatron wavelength downstream. In this section, we develop a simple model of such a feedback system, which finally will allow us to predict the level of the residual emittance growth in the accelerator. 
We consider here a model of an idealized feedback system with a sufficiently broad frequency band to resolve the motion of each bunch in the machine. Let $X_{1}$ denote the beam displacement at the position of the pickup electrode (point 1). The kicker, located a quarter of the betatron wavelength downstream (point 2), deflects the beam by an angle $\alpha$,

$$
\alpha=g \frac{X_{1}}{\sqrt{\beta_{1} \beta_{2}}}
$$

where $\beta_{1}$ and $\beta_{2}$ are the values of the beta function at the positions of the pickup and the kicker electrodes and $g$ is the dimensionless amplification factor (gain) of the feedback system. Using the definition of momentum $p$ according to Equation (1), one can find the change in the momentum $\Delta p_{2}$, produced by the kick:

$$
\Delta p_{2}=\sqrt{\beta_{2}} \alpha=g \frac{X_{1}}{\sqrt{\beta_{1}}}=g x_{1} .
$$

Because the points 1 and 2 have a $90^{\circ}$ phase difference, $x_{1}=-p_{2}$, we can express $\Delta p_{2}$ in terms of the beam momentum at point 2 :

$$
\Delta p_{2}=-g p_{2} .
$$

Since the kicker does not perturb the beam coordinate $x_{2}$, it is easy to write down, in matrix notation, the transformation of the variables $x$ and $p$ resulting from passing through the feedback system:

$$
\left(\begin{array}{c}
x_{2}^{\prime} \\
p_{2}^{\prime}
\end{array}\right)=F\left(\begin{array}{l}
x_{2} \\
p_{2}
\end{array}\right)=\left(\begin{array}{cc}
1 & 0 \\
0 & 1-g
\end{array}\right)\left(\begin{array}{l}
x_{2} \\
p_{2}
\end{array}\right),
$$

where $x_{2}$ and $p_{2}$ refer to the initial and $x_{2}^{\prime}$ and $p_{2}^{\prime}$ the final states of the beam, and $F$ is the transformation matrix. To obtain a complete transformation $M$ that includes the beam motion along the ring outside the feedback system, one has to combine the transformation in Equation (33) with that of Equation (4), multiplying the matrix $F$ by the standard rotation matrix $R$ :

$$
M=R F=\left(\begin{array}{cc}
\cos \mu & \sin \mu \\
-\sin \mu & \cos \mu
\end{array}\right)\left(\begin{array}{cc}
1 & 0 \\
0 & 1-g
\end{array}\right)=\left(\begin{array}{cc}
\cos \mu & (1-g) \sin \mu \\
-\sin \mu & (1-g) \cos \mu
\end{array}\right) .
$$

The influence of the feedback on the beam motion is characterized by the eigenvalues $\lambda_{1,2}$ of the matrix $M$. A simple calculation yields

$$
\lambda_{1,2}=\left(1-\frac{1}{2} g\right) \cos \mu \pm i \sqrt{(1-g) \sin ^{2} \mu-\frac{g^{2}}{4} \cos ^{2} \mu} .
$$


Analysis shows ${ }^{8}$ that $\left|\lambda_{1,2}\right|<1$ only if $g<2$. That means that the feedback system damps oscillations for $g<2$ and would amplify them for $g>2$. If he gain $g$ satisfies the inequality

$$
\frac{g}{\sqrt{1-g}}<2 \tan \mu
$$

both eigenvalues have equal modula smaller than $1:\left|\lambda_{1}\right|=\left|\lambda_{2}\right|=\sqrt{1-g}<1$.

The damping decrement $\gamma$ in the general case can be found as

$$
\gamma=f_{0} \min \left\{\left(1-\left|\lambda_{1}\right|\right),\left(1-\left|\lambda_{2}\right|\right)\right\},
$$

where $f_{0}$ is the revolution frequency. In the limit $g \ll 1$, this gives

$$
\gamma \approx \frac{1}{2} f_{0} g .
$$

Remembering that the beam decoheres on a time scale $\tau_{d}=\left(f_{0} \Delta \nu_{\mathrm{rms}}\right)^{-1}$, in order for the feedback system to suppress the beam oscillations before they decohere, we have to require that the decrement $\gamma$ be much larger than the inverse decoherence time $\tau_{d}^{-1}$; that is,

$$
g \gg \Delta \nu_{\mathrm{rms}}
$$

\subsection{Suppression of Emittance Growth by a Feedback System}

A feedback system is able to damp a single noise kick, but for steady-state noise producing many kicks on each turn of the beam, a residual level of the oscillations still survives. These residual oscillations, combined with the decoherence, cause remittance growth, although with a growth rate lower than the original growth rate given by Equation (19).

A key problem for the theory of feedback is the prediction of the residual emittance growth of the beam in the accelerator. We will calculate it using a simple model, considering first the motion of the beam particles after a single noise kick that displaces the beam distribution function as a whole by $\Delta x, \Delta p$ in the phase plane. Without the feedback, decoherence eventually translates this initial displacement into the incremental increase of the emittance, $\Delta \varepsilon_{0}=\left(\Delta x^{2}+\Delta p^{2}\right) / 2$. Below, we will calculate the increase of the emittance with the feedback.

Denote the coordinate of the centroid of the beam by $\bar{x}$ and the averaged momentum of the beam by $\bar{p}$. In the absence of feedback, they satisfy the following equation,

$$
\frac{d \bar{x}}{d \theta}=\bar{p}, \quad \frac{d \bar{p}}{d \theta}=-\bar{x} .
$$

Equation (38) describes the betatron oscillations of the centroid, neglecting the effect of decoherence.

Assuming $g \ll 1$, it follows from Equation (36) that the damping occurs on a time scale of many turns around the ring. In this case, instead of considering the 
transformation with the matrix $M$, we adopt an approach based on a differential equation for $\bar{x}$ and $\bar{p}$. The feedback adds kicks according to Equation (32) to the motion described by Equations (38). In the limit of small $g$, these kicks must be added to the second part of Equation (38) as follows:

$$
\frac{d \bar{p}}{d \theta}=-\bar{x}-g \bar{p} \tilde{\delta}(\theta)
$$

where the periodic delta function,

$$
\tilde{\delta}(\theta)=\sum_{n=-\infty}^{\infty} \delta(\theta-\mu n),
$$

accounts for periodicity of the kicks. Neglecting higher-order harmonics produced by the kicks, we will average the $\tilde{\delta}$ function over $\theta$ and keep only the time-independent component,

$$
\tilde{\delta}(\theta) \rightarrow \frac{1}{\mu}
$$

With this substitution, combining the first of Equation (38) with Equation (39) yields

$$
\frac{d^{2} \bar{x}}{d \theta^{2}}+\frac{g d \bar{x}}{\mu d \theta}+\bar{x}=0
$$

This is the equation of a damped oscillator. Since we are assuming that $g \ll 1$ and hence $g / \mu \ll 1$, we can find an approximate solution of Equation (42) in the following form:

$$
\bar{x}=e^{-g \theta / 2 \mu}(\Delta x \cos \theta+\Delta p \sin \theta), \quad \bar{p}=e^{-g \theta / 2 \mu}(-\Delta x \sin \theta+\Delta p \cos \theta),
$$

where $\Delta x$ and $\Delta p$ are the initial values of $\bar{x}$ and $\bar{p}$, respectively, produced by a noise kick. These equations demonstrate that an initial perturbation exponentially dies out with the decrement equal to that obtained from the matrix analysis, Equation (36).

Now when we have found the dependence $\bar{p}$ versus $\theta$ and know the interaction with the feedback system, we can solve for the motion of each particle of the beam. To this aim, we will solve the equation of motion for a particle having tune which differs slightly (by $\Delta \nu$ ) from the tune $\nu$ of the centroid of the beam. In our notation, these equations have the forms

$$
\frac{d x}{d \theta}=p, \frac{d p}{d \theta}=-\left(1+\frac{\Delta \nu}{\nu}\right)^{2} x-g \bar{p} \tilde{\delta}(\theta) .
$$

In the limit $g \rightarrow 0$, they govern the motion of a linear oscillator with the tune $\nu+\Delta \nu$, and the $g$ term accounts for the interaction with the feedback. By analogy to Equation (42), using Equations (43) and (44) one finds 


$$
\frac{d^{2} x}{d \theta^{2}}+\left(1+\frac{\Delta \nu}{\nu}\right)^{2} x=-\frac{g}{\mu} e^{-g \theta / 2 \mu}(-\Delta x \sin \theta+\Delta p \cos \theta) .
$$

Integrating this equation with the initial values

$$
x(t=0)=x_{0}+\Delta x, \quad p(t=0)=p_{0}+\Delta p,
$$

where $x_{0}$ and $p_{0}$ give the position of the particle in the phase plane before the displacement, one finds the following result in the limit of large $\theta$ :

$$
x=\left(x_{0}+\frac{4 \pi \Delta \nu}{g} \Delta p\right) \cos \left[\left(1+\frac{\Delta \nu}{\nu} \theta\right)\right]+\left(p_{0}-\frac{4 \pi \Delta \nu}{g} \Delta p\right) \sin \left[\left(1+\frac{\Delta \nu}{\nu}\right) \theta\right] .
$$

From these equations we see that if $\Delta \nu \neq 0$, the feedback does not restore the initial values $x_{0}$ and $p_{0}$ that the particle had before the noise kick, but rather changes them slightly. This residual perturbation will eventually evolve, on a time scale associated with the decoherence process, to an incremental increase of the emittance $\Delta \varepsilon$. To find $\Delta \varepsilon$, one has to average $\left(x^{2}+p^{2}\right) / 2$ over the particle distribution function. In doing so, one finds that the linear terms in $\Delta x$ and $\Delta p$ cancel, and the result is

$$
\Delta \varepsilon=\frac{16 \pi^{2} \overline{\Delta \nu^{2}}}{g^{2}} \Delta \varepsilon_{0}
$$

where $\Delta \varepsilon_{0}=\left(\Delta x^{2}+\Delta p^{2}\right) / 2$ is the emittance increase that would occur if there were no feedback in the system.

After we have considered one kick and shown that the increase of the emittance with the feedback is suppressed according to Equation (47) we can generalize this result for many independent uncorrelated kicks. If Equation (37) holds - that is, the damping time is much smaller than the decoherence time - one can consider that the feedback system reacts independently to each noise kick on the beam. In this case, increases in the emittance due to single noise kicks are simply summed up, giving the following emittance growth formula:

$$
\frac{d\langle\varepsilon\rangle}{d t}=\frac{16 \pi^{2} \overline{\Delta \nu^{2}}}{g^{2}}\left(\frac{d\langle\varepsilon\rangle}{d t}\right)_{0},
$$

where $(d\langle\varepsilon\rangle / d t)_{0}$ is given by Equation (19).

Now let us add the effect of the errors associated with the measurements of the position of the beam. For a wideband feedback system, one can expect these errors to be white noise with the mean square equal to $X_{\text {noise }}^{2}$. This noise generates additional kicks on the beam according to Equation (31):

$$
\Delta p_{\text {noise }}^{2}=\frac{g^{2}}{\beta_{1}} X_{\text {noise }}^{2}
$$




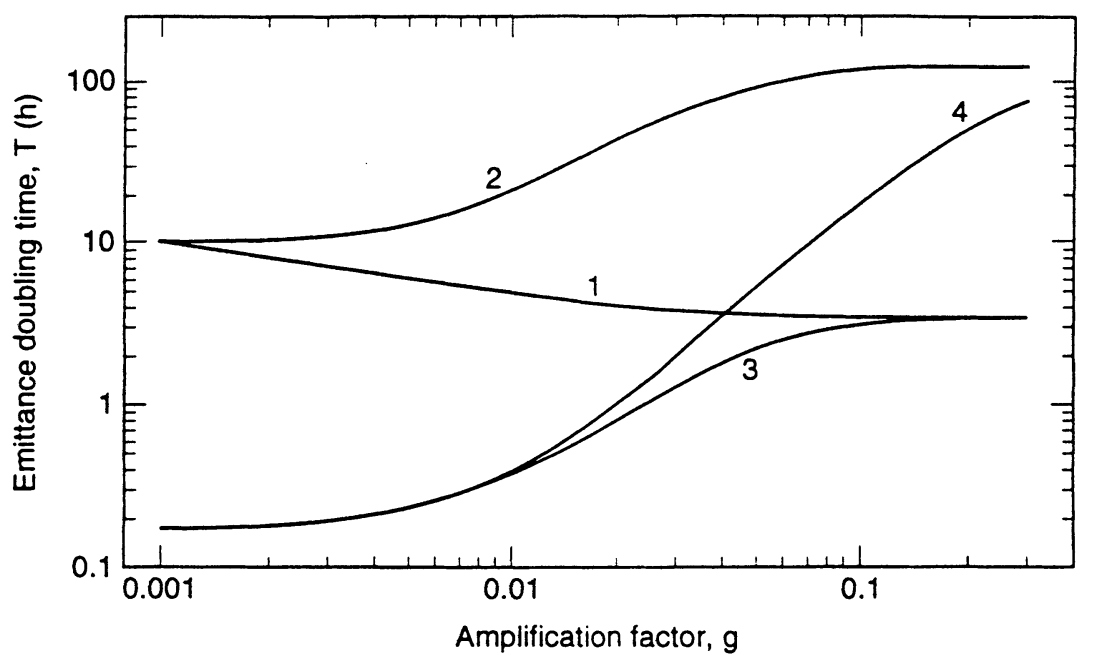

FIGURE 2: Emittance doubling time $T$ as a function of the feedback gain $g$, for different values of $T_{0}$ and $X_{\text {noise }}: 1-T_{0}=10 \mathrm{~h}, X_{\text {noise }}=3.5 \mu m ; 2-T_{0}=10 \mathrm{~h}, X_{\text {noise }}=0.5 \mu m ; 3-T_{0}=10 \mathrm{~min}, X_{\text {noise }}=3.5 \mu \mathrm{m} ; 4-$ $T_{0} \min , X_{\text {noise }}=0.5 \mu \mathrm{m}$. Other parameters are: $\beta_{1}=350 \mathrm{~m}, \Delta \nu_{\mathrm{rms}}=1.8 \times 10^{-4}$.

which, in turn, without feedback would cause the emittance growth

$$
\frac{d \varepsilon_{\text {noise }}}{d t}=\frac{f_{0} g^{2}}{2 \beta_{1}} X_{\text {noise }}^{2} \text {. }
$$

With feedback, this term must be added to $(d\langle\varepsilon\rangle / d t)_{0}$ in Equation (48), giving

$$
\frac{d\langle\varepsilon\rangle}{d t}=\frac{16 \pi^{2} \overline{\Delta \nu^{2}}}{g^{2}}\left[\left(\frac{d\langle\varepsilon\rangle}{d t}\right)_{0}+\frac{f_{0} g^{2}}{2 \beta_{1}} X_{\text {noise }}^{2}\right] .
$$

As an illustration of the use of Equation (51), we show in Figure 2 how the emittance doubling time $T=\varepsilon_{0}(d\langle\varepsilon\rangle / d t)^{-1}$ in an accelerator with feedback depends on the level of external noise (measured in terms of the emittance doubling time without feedback, $T_{0}=\varepsilon_{0}(d\langle\varepsilon\rangle / d t)_{0}^{-1}$ and the accuracy of the Beam Position Monitor (BPM). Figure 2 clearly demonstrates that to suppress the emittance growth, one must have good BPM resolution (small values of $X_{\text {noise}}$ ); otherwise turning on the feedback system might even decrease the emittance doubling time in the machine. 


\section{CONCLUSION}

In this paper we presented a derivation and analysis of emittance growth due to external random noise in a hadron accelerator. The theory predicts that the rate of the emittance growth is proportional to the values of this noise spectrum at the betatron sideband frequencies $f_{0}|\nu-n|$. It is important to emphasize here that the mechanism responsible for emittance growth is the tune spread, though formally $\Delta \nu$ does not enter Equation (19).

We also considered the work of the feedback system and derived the residual emittance growth rate. If the gain of the feedback is much larger than the tune spread in the beam, $g \gg \Delta \nu_{\text {rms }}$, one finds a decrease in the growth rate proportional to $g^{2}$ according to Equation (51). In the opposite limit, $g \ll \Delta \nu_{\mathrm{rms}}$, the feedback would not suppress the emittance growth because decoherence proceeds faster than damping. Internal noise in the feedback system has also been included in the theory in terms of the accuracy of the BPM.

\section{REFERENCES}

1. G.E. Fisher and P. Morton, "Ground motion tolerances for the SSC," SSC Laboratory preprint No. SSC-55, 1986 (unpublished).

2. K.-Y. Ng and J. Peterson, "Ground-motion effects on the SSC," SSC Laboratory preprint No. SSC212, 1989 (unpublished).

3. K.-Y. Ng and J. Peterson, "Effects from measured ground motions at the SSC," SSC Laboratory preprint No. SSCL-227, 1990 (unpublished).

4. K.-Y. Ng and J. Peterson, "Emittance growth due to beam motion," SSC Laboratory preprint No.SSCL-441, 1991 (unpublished).

5. K.-Y. Ng, "Emittance growth due to a small low-frequency perturbation, " Fermilab report No. FN575, 1991 (unpublished).

6. L. Michelotty and F. Mills, "Amplitude growth due to random correlated kicks," in Proceedings of the IEEE Particle Accelerator Conference, (Chicago, IL., USA 1989), V.2, p.1394.

7. S.R. Mane and G. Jackson, "Studies and calculations of the transverse emittance growth in highenergy proton storage rings," in Proceedings of IEEE Particle Accelerator Conference, (Chicago, IL., USA) V.3, p.1801.

8. V.A. Lebedev, V.V. Parkhomchuk, V.D. Shiltsev, A.N. Skrinsky, "Suppression of emittance growth caused by mechanical vibrations of magnetic elements in the presence of beam-beam effects in the SSC," Institute for Nuclear Physics (Novosibirsk) report, 1991 (unpublished).

9. G.V. Stupakov, "Emittance growth caused by magnet vibrations in the SSC," SSC Laboratory preprint No. SSCL-516, 1992.

10. Superconducting Super Collider Site-Specific Conceptual Design, SSC Laboratory preprint No. SSCL-SR-1056, 1990 (unpublished).

11. H.K. Given, "Variations in broadband seismic noise at IRIS/IDA stations in the USSR with the implementations for event detection," Bulletin of the Seismological Society of America, 80(6) (1990) p. 2072.

12. B.A. Baklakov et al., "Investigation of seismic vibrations and relative displacement of linear collider VLEPP elements," in Proceedings of the 1991 IEEE Particle Accelerator Conference, (San Francisco, USA, 1991) p. 3273.

13. V.A. Lebedev et al., "Transverse vibrations of electron beam and ground motion measurements at VEPP-3 storage ring," Institute for Nuclear Physics (Novosibirsk) report, Preprint INP 92-39, 1992. 
14. "Summary and presentations of the workshop on vibration control and dynamic alignment issues at the SSC," SSC Laboratory preprint No. SSCL-SR-1185 (1992).

15. V.A. Lebedev and G.V. Stupakov, "Magnetic field perturbation caused by bending vibrations of a quadrupole," SSC Laboratory preprint No. SSCL-580 (1992).

16. A. Chao and D. Douglas, "Preliminary estimate of emittance growth due to position jitter and magnet strength noise in quadrupole and sextupole magnets," SSC Laboratory preprint No.SSC-N-34 (1985).

17. G.V. Stupakov, "Emittance growth caused by sextipole vibrations in the SSC," SSC Laboratory preprint No. SSCL-575 (1992).

\section{APPENDIX}

To calculate the sum

$$
\Re=\sum_{n, m=0}^{N-1} e^{i \omega T(n-m)} \sin \mu(N-n) \sin \mu(N-m)
$$

from Equation (11) in the limit $N \rightarrow \infty$, first we express sine in terms of the exponential and perform the summation:

$$
\begin{aligned}
\Re= & -\frac{1}{4} e^{2 i \mu N} \frac{\left(e^{i(\omega T-\mu)} N-1\right)\left(e^{-i(\omega T+\mu)} N-1\right)}{\left(e^{i(\omega T-\mu)}-1\right)\left(e^{-i(\omega T+u)}-1\right)} \\
& +\frac{1}{4} \frac{\left(e^{i(\omega T+\mu)} N-1\right)\left(e^{-i(\omega T+\mu)} N-1\right)}{\left(e^{i(\omega T+\mu)}-1\right)\left(e^{-i(\omega T+u)}-1\right)}+c . c= \\
& \frac{1}{4} e^{i \mu(N+1)} \frac{\sin 1 / 2 N(\omega T-\mu) \sin 1 / 2 N(\omega T+\mu)}{\sin 1 / 2(\omega T-\mu) \sin 1 / 2(\omega T+\mu)} \\
& +\frac{1}{4}\left(\frac{\sin 1 / 2 N(\omega T+\mu)}{\sin 1 / 2(\omega T+\mu)}\right)^{2}+c . c .,
\end{aligned}
$$

where $c . c$. denotes the complex conjugate. In the limit $N \rightarrow \infty$, the following identities are valid:

$$
\frac{\sin 1 / 2 N \xi}{\sin 1 / 2 \xi} \rightarrow 4 \pi \tilde{\delta}(\xi), \quad \frac{\sin ^{2} 1 / 2 N \xi}{\sin ^{2} 1 / 2 \xi} \rightarrow 2 \pi N \tilde{\delta}(\xi)
$$

where

$$
\tilde{\delta}(\xi)=\sum_{n=-\infty}^{\infty} \delta(\xi-2 n \pi)
$$


Now, note that $\tilde{\delta}(\omega T-\mu) \tilde{\delta}(\omega T+\mu)$ is identically equal to zero, unless $\nu$ is equal to half an integer - possibility excluded for an accelerator. The only term that is left in Equation (A.2) is

$$
\Re=\pi H \tilde{\delta}(\omega T-\mu) .
$$

Putting (A.5) in Equation (11) gives Equations (12) and (13). 